\title{
Im Netz der Dinge. \\ Kulturen des gelehrten Sammelns in der Personalunion
}

\author{
Dominik Collet
}

Frühe Museen haben in den letzten Jahren eine radikale Neuinterpretation erfahren. Wurden sie früher als obskure Sammelsurien betrachtet, interpretiert man sie heute als zentrale Wissensräume. Die neue Kulturgeschichte des Wissens versteht frühneuzeitliche Sammlungen als contact- oder trading-zones, die zentrale Akteure und Ideen trotz großer geographischer Distanzen miteinander in Berührung brachten. Die Exponate dieser Sammlungen lassen sich in diesem Zusammenhang als boundary objects auffassen, die den Austausch über soziale, religiöse, territoriale oder disziplinäre Grenzen hinweg organisierten. ${ }^{1}$ Diese Neubewertung ist heute so populär, dass eine rekonstruierte Kunst- und Naturalienkammer auch im Zentrum des Berliner Humboldtforums, des größten zeitgenössischen Kulturprojekts in Deutschland geplant ist. Sie soll dort andere, offenere Formen des Austauschs zwischen Deutschlands und der Welt illustrieren und materialisieren. ${ }^{2}$

Folgt man dieser Neubewertung, sollten Sammlungen und ihre Objekte auch für den Austausch innerhalb anderer transnationaler Netzwerke, wie etwa der Personalunion von Großbritannien und Kurhannover, ein vielversprechendes Untersuchungsfeld darstellen. Anhand der zwischen den beiden Territorien zirkulierenden Objekte lassen sich möglicherweise intensivere Formen des Austausches beobachten,

1 James Clifford, Museums as Contact Zones, in: Ders. (Hg.), Routes. Travel and translation in the late twentieth century, Cambridge, Mass. 1997, S. 188-219; Peter Galison, Image \& Logic. A Material Culture of Microphysics, Chicago 1997; Susan Starr / James R. Griesemer, Institutional Ecology, Translations and Boundary Objects. Amateurs and Professionals in Berkeley's Museum of Vertebrate Zoology, 1907-39, in: Social Studies of Science 13 (1989), S. 387-420; Daniela Bleichmar (Hg.), Collecting across cultures. Material exchanges in the early modern Atlantic world, Philadelphia 2011.

2 Vgl. zu der „Kunstkammer“ im Berliner Humboldtforum und der von ihr erhofften Umdeutung der früheren Berliner Kolonialmuseen in „Weltmuseen“: Friedrich von Bose, The making of Berlin's Humboldt-Forum. Negotiating history and the cultural politics of place, in: Darkmatter 11 (2013). http:// www.darkmatter101.org/site/category/journal/issues/11-afterlives/ (zuletzt aufgerufen am 1.11.2013). Für einen weniger politisierten, britischen Versuch, museale Objekte als Medien des globalen Wissenstransfers zu befragen, vgl. Neil MacGregor, A history of the world in 100 objects, London 2010. 
als sie von der politischen Ebene bekannt sind. Zudem werden eventuell auch jene bisher kaum erfassten Transfers sichtbar, die vom kleineren Landesteil Hannover aus in die britische Zentrale führten, wenn man das Untersuchungsfeld von Hof und Administration auf den lebhafteren Bereich von materieller Kultur und Wissen(schaft) erweitert. ${ }^{3}$

Allerdings verdeckt die neue Begeisterung für alte Sammlungen leicht, dass auch objektzentrierte Austauschprozesse mit gravierenden Herausforderungen einhergingen - insbesondere dann, wenn sie in den stratifizierten Gesellschaften des 17. und 18. Jahrhunderts stattfanden. Das Sammeln stellte eine außergewöhnlich gesellige Wissenspraxis dar. Es brachte notwendigerweise eine breite Gruppe von Agenten, Händlern, Informanten, Reisenden und Besuchern miteinander in Kontakt. Die Beteiligung solch unterschiedlicher Akteure besitzt sicherlich das Potential, unser Verständnis darüber zu erweitern, wer in der Personalunion neben dem Hofstaat die Träger von Austauschprozessen waren. Das Aufeinandertreffen einer derart heterogenen Gruppe von Akteuren barg aber auch ein hohes Konfliktpotential. Hinzu kommt, dass der Austausch im musealen Raum nicht nur die Übersetzung zwischen unterschiedlichen sozialen Sphären nötig machte, sondern auch eine Übersetzung zwischen unterschiedlichen Medien: Objekten, Bildern und Texten. Solche komplizierten Transferprozesse laden regelmäßig zu Verzerrungen und Voreingenommenheit ein. Deshalb möchte ich den vielen Formen von Kulturkontakten innerhalb der Personalunion eine weitere, oft unterschätzte Variante hinzufügen: die des ,kreativen Missverständnisses‘.

Da Museen schon ihrer Funktion und Intention nach konservative Gebilde darstellen, setzt die Untersuchung bereits in der Phase vor der Personalunion (1714-1837) ein. Dieser Rückgriff erlaubt es, die Potentiale und Probleme des kulturellen Austausches im musealen Raum anhand von zwei eng miteinander verbundenen Fallstudien zu illustrieren: der Londoner Royal Society des späten 17. Jahrhunderts und der Wiederholung und Emulation des Londoner Projektes durch Göttinger Akademiker während der Personalunion einhundert Jahre später. Beide Beispiele fügen sich nur bedingt in die Erfolgsgeschichte der musealen contact zone. Stattdessen illustrieren sie, dass für historische Austauschprozesse soziale Distanz oftmals von größerem Gewicht

3 Jüngere Forschungen zur Hanoverian dimension britischer Politik haben die Deutung der Union als weitgehend folgenlose dynastische Zwecklösung hinterfragt und sie stattdessen in die europäische Tradition des composite state eingeordnet. Vgl. etwa: Brendan Simms / Torsten Riotte (Hg.), The Hanoverian Dimension in British History 1714-1837, Cambridge 2007; Jeremy Black, Continental Commitment. Britain, Hanover and interventionism 1714-1793, London / New York 2005; Stephen Conway, Continental Connections. Britain and Europe in the Eighteenth Century, in: History 90 (2005), S. 353-374; Nicholas B. Harding, Hanover and the British Empire 1700-1837, London 2007. Diese Neubewertungen sind eng mit der lebhaften Forschung zu interkulturellem Austausch und histoires croisées in transnationalen Netzwerken verbunden, bleiben aber weitgehend auf das Feld der Politik beschränkt. Für eine breitere Anwendung dieser Konzepte auf die Personalunion vgl. Steffen Hölscher / Sune Schlitte (Hg.), Kommunikation im Zeitalter der Personalunion (1714-1837). Prozesse, Praktiken, Akteure, Göttingen 2014. 
war als die geographische Entfernung und dass die Wissenspraktiken, die solche Austauschprozesse flankierten, oft nicht in direkten Transfers resultierten, sondern in unintendierten, aber deshalb nicht weniger wirksamen Formen des Austausches.

\section{Ein Empire der Dinge. Das Museum der Royal Society}

Sammler und klassische Gelehrte verstanden einander über lange Zeit nicht als Partner, sondern als Gegenspieler. Während des 17. Jahrhunderts war das Sammeln häufig durch eine kritische Haltung zur universitären Gelehrsamkeit motiviert und wurde von sozialen Gruppen außerhalb der klassischen Bildungstraditionen getragen. Virtuosi und Connaisseurs verstanden das Sammeln als einen Raum der Freundschaft, der sich durch Konsens und Geselligkeit deutlich von den notorischen Streitereien an den Universitäten abhob. Umgekehrt betrachteten viele Professoren die Aktivitäten der Sammler als naiv und oberflächlich und beklagten deren Bereitschaft Form über Substanz und visuellen Reiz über gründliche Analysen zu stellen. Dass diese scharfen Grenzziehungen zumeist nur in der Imagination der beteiligten Akteure existierten und tatsächlich zahlreiche persönliche und institutionelle Überschneidungen bestanden, bedeutet nicht, dass sie keine praktischen Auswirkungen besaßen. Wie die folgenden Fallbeispiele zeigen, barg ihre Überschreitung für die Grenzgänger ein durchaus reales Risiko, das durch eine ausgeprägte ,soziale Geschmeidigkeit' der Vermittler aufgefangen werden musste.

Als die Royal Society for the Improving of Natural Knowledge 1660 in London gegründet wurde, standen ihre Mitglieder fest auf der Seite der Sammler. Im Anschluss an Francis Bacon favorisierten sie direkte Beobachtung anstelle philosophischer Spekulationen und überliefertem Textwissen. Die Errichtung eines Museums stand daher ganz oben auf ihrer Agenda. Sie verstanden die Sammlung als zentrales Instrument, um eine neue Wissenschaft zu begründen, die sich auf materielle Fakten anstatt auf philosophische Gedankenspiele gründen sollte. Sie hofften zudem, dass die physische Präsenz der realen Objekte helfe, jene Streitereien zu vermeiden, welche die Wissenschaft ihrer Zeit und die englische Nach-Bürgerkriegsgesellschaft insgesamt prägten. ${ }^{4}$

Die ersten Fellows hegten die ambitionierte Vorstellung, ihre Sammlung als ,Kontaktraum' zu nutzen, in dem unmittelbar, ungefilterte Beobachtungen möglich wären - ein Unterfangen, das sich explizit gegen die paper techniques der zeitgenössischen Wissenschaft richtete. Der Kurator der Objekte, Robert Hooke, verkündete programmatisch: Bücher seien

4 Michael Hunter, Science and the shape of orthodoxy. Intellectual change in late seventeenth-century Britain, Woodbridge 1995, S. 135-150; Dominik Collet, Die Welt in der Stube. Begegnungen mit Außereuropa in Kunstkammern der Frühen Neuzeit, Göttingen 2007, S. 269-314. 
for the most part [so] superficial, and the Descriptions so ambiguous, that they create a very imperfect Idea of the true Nature and Characteristick of the thing described [...]. It were therefore much to be wisht for and indeavoured that there might be and kept in some repository as full and compleat a Collection of all varieties of Natural Bodies as could be obtain'd, where an Inquirer might be able to have recourse, where he might peruse, and turn over, and spell, and read the Book of Nature, and observe the Orthography, Etymologia, Syntaxis, and Prosodia of Natures Grammar [...].5

Hookes Vision bestand darin, die Praktiken des Lesens in Büchern auf das Lesen der Natur zu übertragen. Sein Ziel war es, die etablierten Texte seiner Zeit im Museum kritisch zu überprüfen und mit aus direkten Observationen gewonnenem Wissen zu ersetzen - ein Ansatz, der sich auch im stolzen Motto der Gesellschaft „nullius in verba" widerspiegelte.

Solch einen object turn in die Praxis umzusetzen, erwies sich allerdings als äußerst kompliziert. 1660, als die Fellows sich um den Aufbau einer Sammlung bemühten, war England aufgrund des Bürgerkrieges lange Zeit vom blühenden Sammlungswesen auf dem Kontinent abgeschnitten gewesen. Den Grundstock für ihr Museum mussten sie daher von einem deutschen Entrepreneur namens Robert Hubert erwerben. Hubert hatte seine Raritäten zunächst an wechselnden Orten im deutschsprachigen Raum gegen Entrichtung eines Eintrittsgeldes präsentiert, war nach dem 30jährigen Krieg aber nach England übersiedelt, wo er in London ein aufgeschlossenes und zahlungskräftiges Publikum für das hier noch kaum bekannte Medium des Museums gefunden hatte. ${ }^{6}$ Auf dem Kontinent blickten solche Sammlungen bereits auf eine über einhundertjährige Geschichte zurück und hatten zahlreiche eigene Traditionen entwickelt, darunter einen ausgefeilten Kanon sammelnswerten Materials. Nach dem Ankauf von Huberts populärer Sammlung fanden die Fellows daher an Stelle der erwarteten Materialien vor allem wundersame Kuriositäten vor, darunter die Rippe eines „Meermanns“, die Knochen eines „syrischen Riesen“ oder ein Nest of a Bird made like the secret parts of a man. ${ }^{7}$ Diese Raritäten konfrontierten ihre hoffnungsvollen Betrachter unmittelbar mit der komplizierten Aufgabe Objekte zu lesen, die ihres ursprünglichen Kontextes vollständig entkleidet worden waren. Die Fellows entschlossen sich daher notgedrungen, den Erwerb der Objekte selbst in die Hand zu nehmen. Dazu griffen sie auf ein bewährtes Mittel zurück, um Wissenschaft

5 Richard Waller (Hg.), The posthumous works of Robert Hooke. Containing his Cutlerian lectures and other discourses, read at the meetings of the illustrious Royal Society, London 1705, S. 365.

6 Zu Huberts deutschem Hintergrund, vgl. Collet, 2007 (wie Anm. 4), S. 278.

7 Robert Hubert, A Catalogue of Many Natural Rarities [...], London 1664, S. 1, 8 und 26. 
über große Distanzen hinweg zu betreiben: den Fragebogen. ${ }^{8}$ In einem ambitionierten Unternehmen wandten sie sich an renommierte Persönlichkeiten in der ganzen bekannten Welt und schickten ihnen ausführliche Inquiries. Darin listeten sie die Objekte mitsamt den begleitenden Informationen auf, die sie sich aus den jeweiligen Regionen wünschten. Die Verantwortung für dieses anspruchsvolle Projekt übertrugen die Fellows einem weiteren Deutschen, dem Sekretär der Gesellschaft Henry Oldenburg. Er exzerpierte lange Listen von interessanten Objekten aus Reiseberichten, übersetzte sie in verschiedene Sprachen, druckte sie in den Philosophical Transactions, koordinierte ihre Verteilung und sammelte den Rücklauf ein. Nahezu einhundert dieser Listen haben sich erhalten. Sie gingen an respektable Kontaktpersonen in den britischen Kolonien und die Stützpunkte anderer europäischer Staaten in Übersee. In wenigen Jahren gelang es Oldenburg von London aus ein einzigartiges weltumspannendes Netzwerk aufzubauen. ${ }^{9}$

Angesichts der schwierigen politischen und logistischen Bedingungen zur Mitte des 17. Jahrhunderts stellt schon allein diese Tatsache eine bemerkenswerte Leistung dar. Allerdings fand ein über den bloßen Kontakt hinaus gehender Austausch nur sehr begrenzt statt. Die Anzahl der Objekte, die das Londoner Museum erreichten, blieb gering. Zumeist bestanden die Sendungen nur aus tendenziös ausgewählten, fragmentarischen Stücken ohne nennenswerte Dokumentation. Auch die begleitenden schriftlichen Auskünfte halfen kaum, diese Defizite aufzufangen. Die Korrespondenten orientierten sich am etablierten Kanon sammelnswerter Objekte. Sie konzentrierten sich auf bizarre Stücke, die die Andersartigkeit der fernen Welten illustrierten und verwiesen darauf, dass die begleitenden Informationen aus gelehrten Büchern kompiliert werden könnten, deren Autorität sie weit höher einschätzten als ihre eigene. Die so übermittelten Exotika verweisen darauf, dass die Londoner und ihre Korrespondenten nicht nur eine räumliche sondern auch eine soziale Distanz trennte. Die wissenschaftlichen Codes der Sammler und ihrer Zulieferer stammten aus ganz unterschiedlichen Lebenswelten. ${ }^{10}$

Einige Korrespondenten wussten diese Distanz gezielt zu instrumentalisieren. Als Philippo Vernatti, ein Faktor der niederländischen Ostindienkompanie, die Fragen der Londoner erhielt, antwortete er mit etablierten Kuriositäten - „Steine“ aus Schlangenköpfen, die wirksam gegen Gifte seien, ostindisches „Stinkholz“, das die

8 Zum Gebrauch des Fragebogens als Kontroll- und Disziplinierungsinstrument, vgl. Arndt Brendecke, Empire und Empirie. Funktion des Wissens in der spanischen Kolonialherrschaft, Köln / Weimar / Wien 2009, S. 270-290 sowie Joan-Pau Rubiés, Instructions for travellers. Teaching the eye to see, in: History and Anthropology 9 (1996), S. 139-190.

9 Collet, 2007 (wie Anm. 4), S. 281-298. Die Listen finden sich heute in den Royal Society Archives, London, CP XIX "Questions and Answers".

10 Dominik Collet, Big sciences, open networks, and global collecting in early museums, in: David Livingstone / Peter Meusburger (Hg.), Geographies of science, Dordrecht 2010, S. 121-138. 
Wilden für heidnische Beschwörungen verwendeten oder an männliche Genitalien erinnernde Vogelnester, die von Chinesen angeblich als Aphrodisiakum genutzt würden. Ging es hingegen um Objekte und Informationen über Handelsgüter, Seekarten oder Bodenschätze, blieb Vernatti bemerkenswert wortkarg. Wie die meisten Korrespondenten war er sich der engen Verbindung von Empire und Empire genau bewusst. Seine Strategie suggestiv (re-)kontextualisierte Objekte als Täuschkörper einzusenden, um die Fellows von sensibleren Themen abzulenken, erwies sich als ausgesprochen erfolgreich. Vernattis faszinierende „Informationen“ und seine Exponate wurden von der Royal Society umgehend als vorbildhaft veröffentlicht. ${ }^{11}$

Zuletzt scheiterten auch noch die Versuche der Fellows, die eingesandten Objekte zur Grundlage experimenteller Überprüfungen zu machen. So hatte Vernatti zwar einen schillernden Bericht und eine Probe des berüchtigten Makassar-Giftes eingesandt - ein Gift von so außergewöhnlicher Potenz, dass es angeblich nur durch die rasche Einnahme der eigenen Exkremente behandelt werden konnte. ${ }^{12}$ Alle Versuche der Fellows, die Wunder-Substanz an Katzen und Hunden zu testen, verliefen jedoch negativ. Angesichts der schlechten Dokumentation der Proben und der wachsenden Zahl von einander bestätigenden Reiseberichten, sahen sich die Londoner aber nicht in der Lage, die Geschichte tatsächlich zu widerlegen. Anstatt falscher Texte vermuteten sie vielmehr das „falsche "Objekt vor sich zu haben. ${ }^{13}$ Für den Austausch valider Informationen erwiesen sich die dekontextualisierten Objekte als ebenso ungeeignet wie der Kontakt zwischen Akteuren aus unterschiedlichen sozialen Kontexten.

Schließlich fand sich die Gesellschaft mit dem stolzen Motto „Nach niemandes Worten“ damit ab, in ihren Veröffentlichungen Informationen zu präsentieren, die aus genau jenen Texten kompiliert waren, die sie eigentlich hatte überprüfen wollen. Das Unternehmen kulminierte in Werken wie der voluminösen History of Plants, einem Werk von immenser Gelehrsamkeit, dessen vier gewaltige Foliobände aus nichts als Text bestanden. Eine vollständige, aber kaum nutzbare Übersetzung von Objekten in Schrift. ${ }^{14} \mathrm{Zu}$ Beginn des 18. Jahrhunderts wurde die Hoffnung, die Sammlung als effektives Vehikel gelehrten Austausches zu nutzen, stillschweigend aufgegeben. Das Sammeln hatte sich als big science erwiesen: als Unternehmen, das die Zusammenarbeit einer Vielzahl von Akteuren über gewaltige geographische und soziale Distanzen hinweg nötig machte. Solch ein Unterfangen harmonierte nur schlecht

11 Vgl. Collet, 2010 (wie Anm. 10), S. 126 f. sowie Thomas Sprat, The History of the Royal Society of London. For the Improving of Natural Knowledge, London 1667, S. 158-172.

12 Vgl. Daniel Carey, The political economy of poison. The kingdom of Makassar and the early Royal Society, in: Renaissance Studies 17 (2003), S. 517-543.

13 Anon., On the nature of a certain stone, found in the Indies, in the head of a serpent, in: Philosopical Transactions of the Royal Society 1 (1665), S. 102f. und Collet, 2007 (wie Anm. 4), S. 303-305.

14 John Ray, Historia plantarum species hactenus editas aliasque insuper multas noviter inventas $\&$ descriptas complectens [...], 3 Bde, London 1686-1704. 
mit einer Wissenspraxis, die auf engem persönlichen Kontakt, vertrauenswürdigen Zeugen sowie dem sozialen Status gründete, der aus finanzieller Unabhängigkeit erwuchs. ${ }^{15}$ Stattdessen konzentrierten sich die Fellows allmählich auf die „kleinen Wissenschaften“, die Mathematik, Physik oder Optik, deren besser nachvollziehbare und wiederholbare Resultate vor Ort, in der technisch und sozial besser kontrollierten Umgebung des Labors, erzielt werden konnten. ${ }^{16}$ Das Museum der Royal Society entwickelte sich statt zum Forschungsraum zum Salon. In London stellte es bald eine zentrale Touristenattraktion dar, die aber in der zunehmend auf experimentelle Verfahren festgelegten Royal Society keinen nennenswerten Platz mehr hatte.

Unglücklicherweise versäumten es die Fellows, die sonst jede ihrer Aktivitäten penibel dokumentierten, diese Entwicklung öffentlich zu machen. Sonst hätten sie die Göttinger Gelehrten des zweiten Fallbeispiels vor mancher Enttäuschung bewahren können. Stattdessen publizierten sie einen sorgfältig redigierten Katalog ihrer Sammlung, der mehreren Gelehrtengenerationen als vermeintliches Vorbild diente. ${ }^{17}$ Viele Ideen der Fellows kehren daher im zweiten Beispiel nahezu wortwörtlich wieder.

\section{Emulation und Innovation. Das Akademische Museum der Universität Göttingen}

Auch die Universität Göttingen, 1737 vom britischen König Georg II. gegründet, verstand sich als Reforminstitution. Die neuartige Kombination der Universität mit einer Akademie der Wissenschaften bezog ihre Inspirationen auch aus dem Erfolg der Royal Society. Wie in London bildete auch in Göttingen eine zentrale Forschungssammlung von Beginn an einen integralen Bestandteil der akademischen Konzeption (Abb. 1+2). ${ }^{18}$ Aufgrund finanzieller Engpässe konnten die ursprünglichen Ideen erst 1773 umgesetzt werden. In diesem Jahr wurde - eine weitere Parallele zu London eine bereits bestehende Privatsammlung erworben. Der Ankauf wurde zudem durch den gemeinsamen Schirmherrn von Royal Society und Göttinger Universität, König

15 Collet, 2010 (wie Anm. 10), S. 129-134.

16 Hunter, 1995 (wie Anm. 4), S. 150 und Palmira Fontes da Costa, The culture of curiosity at the Royal Society in the first half of the eighteenth century, in: Notes and Records of the Royal Society 56 (2002), S. 147-166.

17 Nehemiah Grew, Musaeum Regalis Societatis. Or, a catalogue and description of the natural and artificial rarities belonging to the Royal Society and preserved at Gresham Colledge [...], London 1686.

18 Marie Luise Allemeyer / Dominik Collet / Marian Füssel, The "Academic Museum". Göttingen's university collections as a space of knowledge production and cultural heritage, in: Opuscula Musealia 18 (2010), S. 15-22 sowie zum Museumsgebäude: Gunther Beer, Beitrag zur Baugeschichte des Akademischen Museums 1773 bis 1877 mit drei Gebäudeplänen des Akademischen Museums, in: Museumsbrief 29 (2010), S. $2-20$. 


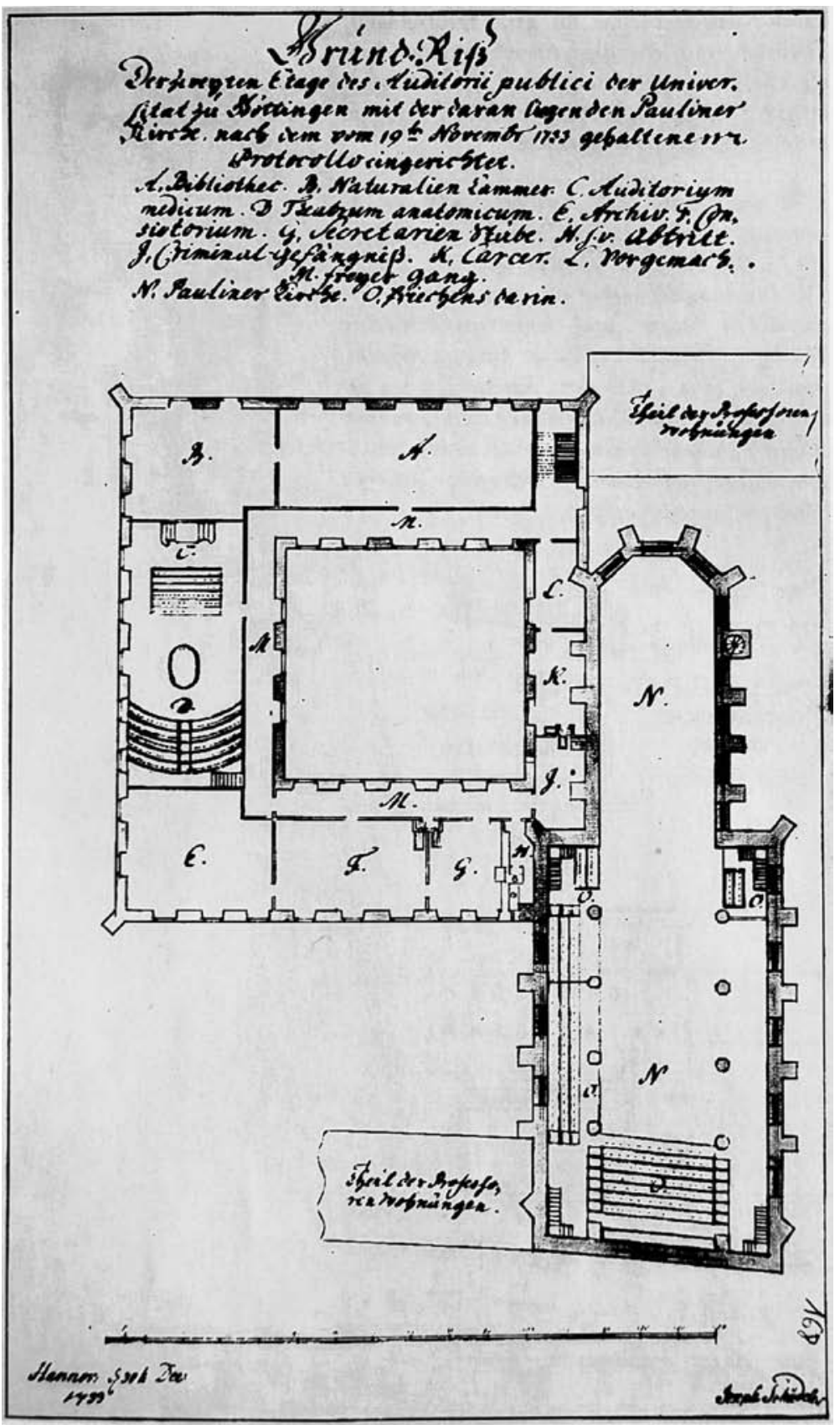

Abb. 1: Bauzeichnung von Joseph Schädeler. Zukünftige Universität in Göttingen mit Raum B „Naturalienkammer“.

NLA Hauptstaatsarchiv Hannover Nr. 23d Göttingen 26pm 


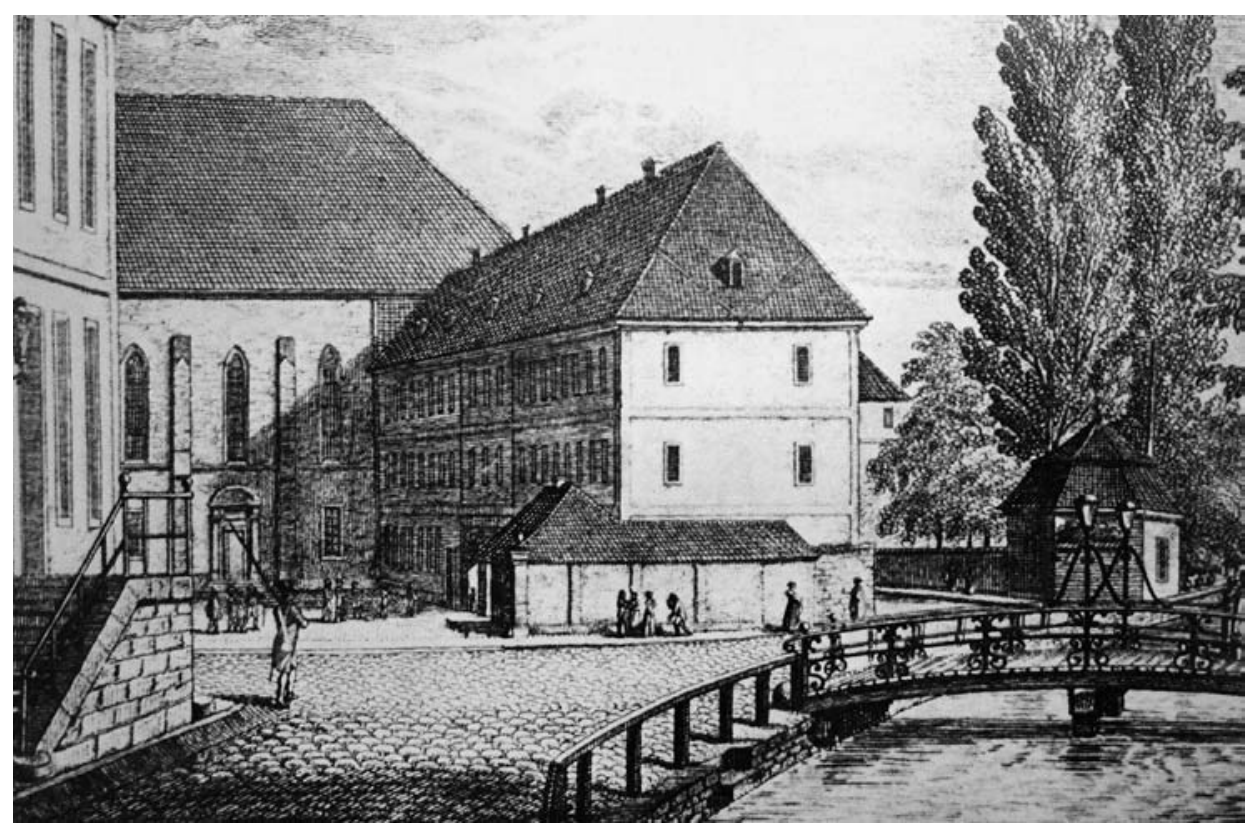

Abb 2: Academisches Museum, Göttingen. Kupferstich um 1800, aus: Alfred Oberndiek, Göttinger Universitätsbauten. Göttingen 1989, S.31

Georg III., finanziert. ${ }^{19}$ Auch die ersten Kommentare der Göttinger Professoren klingen vertraut. Ganz ähnlich wie Robert Hooke verkündete Johann Friedrich Blumenbach, Kurator der Göttinger Sammlung:

Di[e] älteren Sammlungen hatten doch fast durchgehends den Fehler, daß man mehr Seltenheiten als Merkwürdigkeiten der Natur zusammenrafte; [...] Aus dieser letzten Rücksicht werden nun vorzüglich akademische Cabinette wichtig, deren gänzliche Bestimmung dahin abzweckt, dass sie nicht zum Prunck, sondern lediglich zum Gebrauch, zur Untersuchung und zum Unterricht dient [...].

Er fügte stolz hinzu: Göttingen ist die erste Universität in Deutschland, vielleicht in Europa, die mit einem eigentlich akademischen Museum versehen worden; und wir halten uns verpflichtet, von ihm, auch schon als Epochemachenden Phänomen zu sprechen. ${ }^{20}$ Solche Werbeschreiben illustrieren, dass sich mit dem Museum auch noch andere

19 Johann Friedrich Blumenbach, Einige Nachrichten vom academischen Museum zu Göttingen, in: Annalen der Braunschweigisch-Lüneburgischen Churlande 1 (1787), S. 84-99.

20 [Johann Friedrich Blumenbach], Etwas vom Akademischen Museum in Göttingen, in: Georg Christoph Lichtenberg (Hg.), Taschenbuch zum Nutzen und Vergnügen fürs Jahr 1779, Göttingen 1778, S. 45-57, hier S. $47 \mathrm{f}$. 
Hoffnungen verbanden: die Anwerbung vermögender, zumeist adeliger Studierender, die mit den zeitgenössischen Sammlungen gut vertraut waren, sowie die Steigerung des internationalen Renommees der Universität. Sie tragen aber auch der Tatsache Rechnung, dass sich die älteren europäischen Universitäten der objektorientierten Forschung tatsächlich nur langsam und in den Randgebieten ihres Curriculums geöffnet hatten. ${ }^{21}$

Wie die Royal Society erwarben auch die Göttinger Professoren zunächst eine Sammlung, die ihnen in weiten Teilen als planlos und ungeordnet erschien. Es handelte sich um das Kabinett Christian Wilhelm Büttners, über den Goethe später berichtete, dass zu seinen Eigenheiten [...] unbegränzte Neigung zu wissenschaftlichem Besitz, beschränkte Genauigkeitsliebe, und völlige $[\mathrm{r}]$ Mangel an allgemein überschauendem Ordnungsgeiste gehörten. ${ }^{22}$ Auf dieser materiellen Grundlage eigene wissenschaftliche Sammlungspraktiken umzusetzen, erwies sich als äußerst schwierig. Dies galt umso mehr, als die Göttinger Sammlung von Beginn an als öffentliche Institution ausgelegt war und so in einer engen Beziehung zu den fest etablierten Traditionen der privaten Kuriositäten-Liebhaber stand. Auch das Akademische Museum entwickelte sich rasch zu einer zentralen Attraktion für Reisende und zu einer Schnittstelle für den geselligen Austausch von Liebhaberstücken und Kuriositäten - ein Prozess, der eindrucksvoll in einem Besucherbuch dokumentiert ist, das bereits für die erste Dekade über 3.000 Einträge verzeichnet. ${ }^{23}$ Um diesen Entwicklungen zu begegnen, entschlossen sich auch die Göttinger Professoren dazu, Objekte unabhängig vom etablierten Austausch von Kuriositäten zu erwerben. Um dieses Ziel zu erreichen, war die Universität allerdings gezwungen, auf Akteure außerhalb ihrer eigenen Reihen zurückzugreifen Professoren waren für weite und strapaziöse Reisen kaum zu gewinnen. Die Öffnung zur Welt war im allumfassenden Anspruch der Sammlung bereits angelegt. Im universitären Raum aber, in dem Status und Reputation zentrale, beständig umkämpfte Distinktionsmerkmale waren, stellte eine solche personelle und räumliche Ausweitung ein großes Wagnis dar - auch dann, wenn sie sich im privilegierten Rahmen der Personalunion vollzog. Kaum ein Sammler oder Agent teilte die wissenschaftlichen Überzeugungen und Praktiken der Professoren. ${ }^{24}$

21 Marian Füssel, Die Universität der Dinge. Zur universitätshistorischen Verortung des Sammlungswesens, in: Georg-August-Universität Göttingen (Hg.), Dinge des Wissens. Die Sammlungen, Museen und Gärten der Universität Göttingen, Göttingen 2012, S. 52-59.

22 Johann Wolfgang von Goethe, Poetische und prosaische Werke, Bd. 2, Tübingen / Stuttgart 1847, S. 557.

$23 \mathrm{Zu}$ den Besuchern vgl. Christine Nawa, Sammeln für die Wissenschaft. Das Academische Museum Göttingen 1773-1840, M.A. Arbeit, Göttingen 2005, http://webdoc.sub.gwdg.de/master/2010/nawa/nawa. pdf (zuletzt aufgerufen am 1.11.2013).

24 Dominik Collet, Universitäre Sammlungen als contact zone. Gesellige und gelehrte Sammlungspraktiken im Akademischen Museum der Universität Göttingen (1772-1840), in: Traverse 3 (2012), S. 41-52. 
Dieses Konfliktpotential war bereits deutlich geworden, als Albrecht von Haller, Präsident der Göttinger Akademie der Wissenschaften und zugleich auswärtiges Mitglied der Londoner Royal Society, 1752 eine Sammlungsexpedition nach Amerika organisierte hatte. Haller versuchte das Potential der Göttinger Verbindung mit Großbritannien bestmöglich zu nutzen um Naturalien aus allen drey Reichen der Natur zu sammeln. Er sicherte sich die Unterstützung des britischen Königs und verwies ausdrücklich auf das Vorbild der Royal Society. ${ }^{25} \mathrm{Er}$ war sich aber auch der professoralen Vorbehalte seiner Kollegen bewusst. Aus diesem Grund lehnte Albrecht von Haller das Angebot ab, die Sammlungsreise über den Wiener Kaiserhof zu finanzieren. ${ }^{26} \mathrm{Um}$ den betont akademischen Charakter zu wahren, entschied er sich stattdessen für ein Subskriptionsmodell. Dabei gab man wieder an, sich auf englische Vorbilder zu beziehen, die an der britischen Landesuniversität Göttingen besonderes Ansehen genossen:

In England ist mancher wichtige Entwurf durch den Weg der Subscription ausgeführet worden, und so rühmlich dergleichen patriotische Unternehmung dieser großmüthigen Nation sind, eben so viel Ehre wird es jedem Mitgliede dieser Gesellschaft $[\ldots]$ bringen. ${ }^{27}$

Zugleich konnte Haller so an die Erfahrungswelt der Professoren anknüpfen, da ihnen Subskriptionsmodelle aus dem Buchmarkt bereits vertraut waren. Um die textgebundenen Traditionen der Professoren weiter zu bedienen, versprach Haller den Subskribenten neben den Objekten auch exklusive schriftliche Berichte sowie Vorabdrucke etwaiger Reisebeschreibungen. ${ }^{28}$ Ein Risiko musste er in Ermangelung reisewilliger Gelehrter jedoch mit dem Expeditionsleiter eingehen. Angehende Universitätslehrer, wie etwa der junge August Ludwig von Schlözer, standen für ein solch riskantes Unternehmen nicht zur Verfügung. ${ }^{29}$ Hallers Wahl fiel daher auf Christlob Mylius, einen der vielen selbsternannten Experten, die sich im Umfeld der Sammlungen bewegten. Mylius verstand sich allerdings eher auf die Welt der Berliner Salons als auf die Usancen der Universität und sah sich selbst als Journalist, Naturforscher und Dichter.

25 Vgl. die Archivalien zu Hallers Amerika-Expedition, in: Staats- und Universitätsbibliothek Göttingen, Abt. Handschriften und seltene Drucke, 2 H Lit. Biogr. IV 7270.

26 Christlob Mylius, Nachricht von des Herausgebers vorhabenden Reise nach America, in: Physikalische Belustigungen 2 (1773), S. 708-731, hier S. 718. Die Finanzierung sollte unter Vermittlung Gerard von Swietens über das kaiserliche Naturalienkabinett in Wien erfolgen. Möglicherweise spielten auch dynastische Loyalitäten eine Rolle, da das Göttinger Unternehmen bereits von Georg III. unterstützt wurde, wie der Text vermerkt (ebd.).

27 Mylius, 1773 (wie Anm. 26), S. $711 \mathrm{f}$.

28 Mylius, 1773 (wie Anm. 26), S. 711 f. Zu den britischen botanischen Expeditionen, die über Subskriptionen finanziert wurden, vgl. Collet, 2007 (wie Anm. 4), S. 241-243 und 265.

29 Johann Gottlieb Buhle (Hg.), Johann David Michaelis. Literarischer Briefwechsel, 3 Bde, Leipzig 1794-96, hier Bd. 1, S. 192. 
Dies wurde umgehend zu einem Problem, als Mylius während der Reise noch in London verstarb. Verärgerte Professoren begannen umgehend damit, Gerüchte über den zweifelhaften Charakter des Reisenden zu verbreiten. Besonderen Unmut rief dabei die übermäßig lange Zeit hervor, die Mylius in den niederländischen und englischen Amateur-Sammlungen verbracht hatte. Haller selbst befand sich zu dieser Zeit allerdings bereits wieder im heimatlichen Bern und entging so dem Unmut seiner Kollegen. ${ }^{30}$ Vergleichbare chronique scandaleuses finden sich auffällig häufig im Umfeld frühneuzeitlicher Sammlungen. Sie verweisen darauf, dass das Übersetzen zwischen Amateuren und Gelehrten, zwischen unterschiedlichen geographischen Räumen sowie zwischen Objekten und Texten ein heftig umstrittenes Wissenschaftsfeld umriß. ${ }^{31}$

Die Göttinger unternahmen deshalb bei ihrem zweiten Anlauf außergewöhnliche Anstrengungen um ein weiteres Desaster zu vermeiden. Wieder war es einer der ausgesprochenen anglophilen Professoren, von dem die Initiative ausging, nämlich Johann David Michaelis. Als er eine Sammlungsexpedition in den Orient plante, setzte er auf eine lange Reihe von Vorsichtsmaßnahmen. Michaelis wählte potentielle Kandidaten gezielt aufgrund ihrer engen Verbindung mit der universitären Welt aus. Um diese Beziehung noch zu festigen, stellte er sicher, dass die beiden Leiter der Expedition vor ihrer Abreise selbst zu Professoren ernannt wurden. Zudem griff Michaelis, der mit der Royal Society durch seinen Besuch in London 1741 und später auch als Mitglied eng verbunden war, auf das bereits von den Londoner Fellows eifrig benutzte Instrument des Fragebogens zurück. Als die beiden Expeditionsleiter Peter Forskal und Friedrich von Haven aber frühzeitig an Malaria verstarben, löste diese Tragödie umgehend wieder jene persönlichen Angriffe auf die beiden Reisenden aus, die ganz ähnlich schon in Mylius' Fall geäußert worden waren. Die Hoffnung der

30 Vgl. Reimer Eck, Christlob Mylius und Carsten Niebuhr. Aus den Anfängen der wissenschaftlichen Forschungsreise an der Universität Göttingen, in: Göttinger Jahrbuch 34 (1986), S. 11-43, der die Topik der Skandalberichte aber nicht hinterfragt. Zu Mylius' zahlreichen Besuchen von privaten Sammlungen im Tagebuch seiner Reise, vgl. Abraham Gotthelf Kästner, Christlob Mylius, Tagebuch seiner Reise von Berlin nach England 1753. Aus der Handschrift, in: Archiv zur neuern Geschichte, Geographie, Natur- und Menschenkenntnis. Bd. 1 Leipzig 1785, S. 85-167 und Bd. 5, Leipzig 1786, S. 39-140. Zu Mylius' engen Kontakten mit Londoner Virtuosi vgl. seinen aus London an Haller und die Göttinger Akademie gerichteten Artikel: Christlob Mylius, Beschreibung einer neuen Grönländischen Thierpflanze, London 1753. Der Text betonte zwar Mylius' Verbindung zur Royal Society (S. 5), befasste sich aber bezeichnenderweise mit einem liminalen Lebewesen (vermutlich einem Polypen), das mit seinem hybriden Status zwischen Pflanzen- und Tierreich ganz der wissenschaftlichen Episteme der Amateursammler entsprach und von dessen Untersuchung Mylius selbst freimütig bezweifelte, dass sie jemals im Leben einigen Nutzen haben wird (S. 4) - ein Aspekt, der den Göttinger Professoren allerdings besonders wichtig war.

$31 \mathrm{Zu}$ ähnlichen Vorwürfen im Rahmen der Sammlungsreisen Johann Michael Wanslebens in den Orient, Laurentius Rhinhubers nach Äthiopien oder James Reeds, Hugh Jones, William Vernons und David Kriegs nach Amerika, vgl. Collet, 2007 (wie Anm. 4), S. 132-165 und 242ff. Zum Ringen britischer Naturhistoriker wie Joseph Banks und Hans Sloane um Anerkennung in der traditionellen Gelehrtenwelt, vgl. Caspar Hirschi, Men of Sciences versus Macaronies. Die Polemik gegen die Amateur Gentlemen der Royal Society im späten 18. Jahrhundert, in: Frauke Berndt und Daniel Fulda (Hg.), Die Sachen der Aufklärung, Hamburg 2012, S. 193-206. 
Göttinger Gelehrten ruhte nun auf dem letzten Überlebenden, dem Landvermesser und ehemaligen Göttinger Studenten Carsten Niebuhr. Michaelis begann für den Reisenden immer umfassendere Fragebögen zu verfassen, um seine Übersetzung von Natur in Text möglichst umfassend zu kontrollieren und zu disziplinieren. Die sechs bekannten Versionen kulminierten schließlich in einem 400 Seiten umfassenden Fragebogen. Neben seinem nominellen Ziel sollte er auch dazu dienen, sich im Falle weiterer Enttäuschungen vor der europäischen Gelehrtenwelt zu rechtfertigen, weshalb Michaelis seine Fragen an eine Gesellschaft Gelehrter Männer sowohl in deutscher als auch in französischer Sprache drucken ließ. ${ }^{32}$ In seinem Vorwort fasste Michaelis die Schwierigkeiten der disziplinierten Übersetzung von Objekten in Texte folgendermaßen zusammen:

Die Grundlagen der Naturgeschichte, die ich ihr Alphabet nennen möchte, sind doch in der That nur ein Wörterbuch, in eine gewisse Ordnung nach natürlichen Classen gebracht. Daher bestehe ein Mangel darin, daß den Reisenden allein überlassen wird, was sie von auswärtigen Ländern melden sollen, ohne ihnen Fragen vorzulegen und sie zu unterrichten [...]. Sie sehen unzählige Dinge vor Augen, auf die sie nicht merken [dass ein Gelehrter in Europa sie] zu Aufklärung einer Dunkelheit gebrauchen kann.

Dem Professor für orientalische Sprachwissenschaft erschienen die größten Herausforderungen des Wissenstransfers anhand von Objekten dennoch weniger in der geographischen Distanz zu liegen, als darin, Objekte in Text und Amateure in Gelehrte zu verwandeln..$^{33}$

Die professorale Kontrolle forderte - wie schon im Falle der Royal Society einen hohen Preis. Nach seiner Rückkehr benötigte Niebuhr, den die Fragen nach einer abenteuerlichen Reise tatsächlich noch im Feld erreichten, mehr als zehn Jahre, um die Antworten zu verfassen. ${ }^{34}$ Als er sein Werk beendet hatte, ereilte seinen Text ein ähnliches Schicksal wie die History of Lants der Royal Society. Während sein Buch die höchsten Erwartungen erfüllte, endete der Großteil seiner gesammelten

32 Johann David Michaelis, Fragen an eine Gesellschaft Gelehrter Männer die auf Befehl Ihro Majestät des Königs von Dännemark nach Arabien reisen, Frankfurt am Main 1762; Johann David Michaelis, Receuil de Questions, Proposées à une Société de Savans qui par Ordre de sa Majesté Danoise Font le Voyage de l'Arabie, Frankfurt am Main 1763. Eine detaillierte Untersuchung zu den britischen Vorbildern für Michaelis' Fragebogen bietet: Daniel Carey, Arts and Sciences of Travel, 1574-1762. The Arabian Journey and Michaelis's Fragen in context, in: Ib Friis / Michael Harbsmeier / Jørgen Bæk Simonsen (Hg.), Early scientific expeditions and local encounters. New Perspectives on Carsten Niebuhr and The Arabian Journey, Kopenhagen 2013, S. 27-50, hier S. 34-38.

33 Michaelis, 2013 (wie Anm. 32), Vorrede. Michaelis verwies im Vorwort auch auf die Bedeutung von Naturaliensammlungen bzw. „europäischen Cabinettern“ für sein Projekt, gestand aber zugleich, dass seine [a] usfübrlichen Fragen [...] selbst etwas von Antwort enthalten (ebd.).

34 Carsten Niebuhrs Arbeit mündete schließlich in dem vielbeachteten Werk: Beschreibung von Arabien. Aus eigenen Beobachtungen und im Lande selbst gesammelten Nachrichten, Copenhagen 1772. 
Objekte unbeachtet und vernachlässigt im Keller der Kopenhagener Akademie der Wissenschaften. Carl von Linné beklagte sich daher bei Michaelis, die Naturalien selbst seien: mehrentheils ohne gehörige Wartung [geblieben, so] daß der Spiritus verflogen wäre, und dass sie verdürben. Nach ihrer, Übersetzung' in Sprache und ohne ihren ursprünglichen Kontext waren die Dinge stumm und unlesbar geworden. ${ }^{35}$

Mylius' und Niebuhrs Erfahrungen illustrieren die Herausforderungen musealer Kontakte. Solche cultural broker, Vermittler zwischen akademischen und nicht-akademischen Praktiken, zwischen Hannover und dem Empire, zwischen Objekt und Buch begaben sich in ein heftig umstrittenes Feld. Um an Sammlerstücke zu gelangen, mussten sie die Universität verlassen, durften deren soziale und wissenschaftliche Codes jedoch nicht vernachlässigen. Bei Problemen ließ sich ihr Außenseiterstatus leicht gegen sie verwenden, während die erfolgreiche Übersetzung die totale aber nicht immer sinnvolle Übernahme akademischer Wissenspraktiken verlangte. Mylius und Niebuhr stellten dabei keine Einzelfälle dar. Auch berühmte Naturforscher wie Hans Sloane und Joseph Banks, Präsidenten der Royal Society, mussten sich von klassischen Gelehrten aufgrund ihrer als exzentrisch empfundenen Reise- und Sammeltätigkeit weithin als Amateure und Macaronies verspotten lassen (Abb. 3). ${ }^{36}$

Ganz ähnlich wie ihre Londoner Kollegen wurden die Göttinger Professoren durch die Enttäuschungen der Expeditionsunternehmen zu dem Versuch inspiriert, ihre dekontextualisierten Sammlungsobjekte nun aus sich selbst heraus zum Sprechen zu bringen. Da sie für kaum ein Exponat über eine nennenswerte Dokumentation verfügten, erwies sich dies - wie zuvor in London - als ausgesprochen schwierig. Als der dänische König den Göttingern 1781 eine ägyptische Mumie verehrte, bot sich den Professoren eine einmalige Gelegenheit (Abb. 4). Für die überzeugten Aufklärer stellte die Mumie nicht nur ein prominentes Beispiel für zahlreiche Mythen und Aberglauben dar, sie sahen sie auch als Geschenk aus der Welt der sammelnden Amateure und Fürsten. Zwar schätzte man dieses Objekt als Visualisierung der weltweiten Kontakte und der Reputation der Göttinger Universität, zugleich verstand man es aber als Chance, ein Beispiel für eine professionelle Akademisierung vormals aristokratischer Sammlerstücke zu geben. Wie im Falle des Makassar-Giftes kam für die experimentelle Überprüfung des Objektes ein interdisziplinäres Team aus Professoren der Linguistik, Archäologie, Anthropologie und Medizin zusammen, um eine Sektion vorzunehmen, die publizistisch intensiv begleitet wurde. ${ }^{37}$ Die Professoren arbeiteten so gründlich, dass im Inneren der Mumie kaum etwas an seinem Platz blieb. Ihr detailliert veröffentlichter Befund kam allerdings nur zu bescheidenen Er-

35 Michaelis, 1794-1796 (wie Anm. 29), S. 202. Niebuhr schickte auch einige kleinere Objekte an das Göttinger Museum. Eck, 1986 (wie Anm. 30), S. 42.

36 Hirschi, 2012 (wie Anm. 31), S. 193-206.

37 Göttingische Anzeigen von gelehrten Sachen 123 (08.10.1781), S. 985-992. 


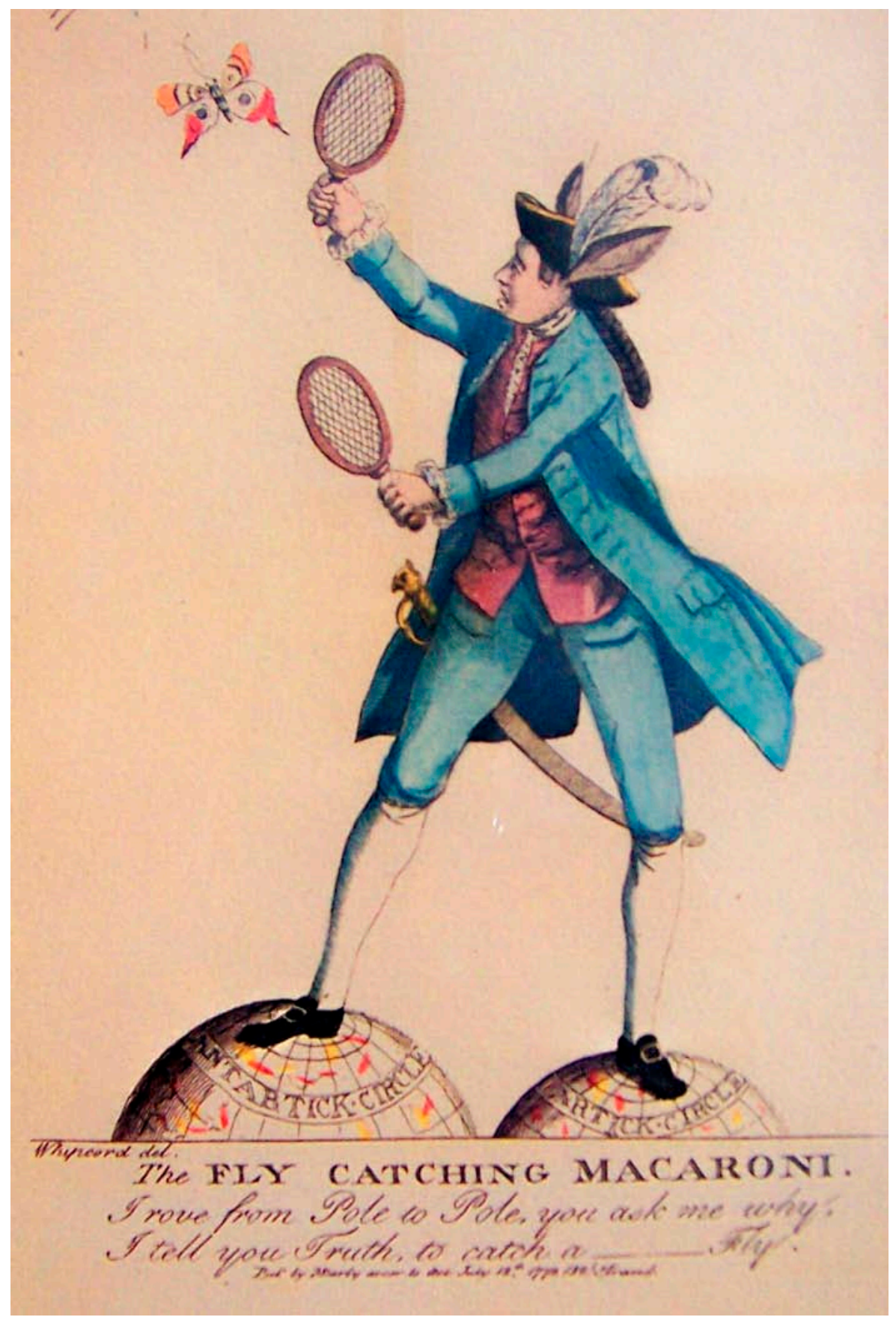

Abb. 3: Karikatur auf Joseph Banks. Kolorierter Kupferstich. M. Darly, The fly catching Macaroni, London 1772. nla.gov.au 


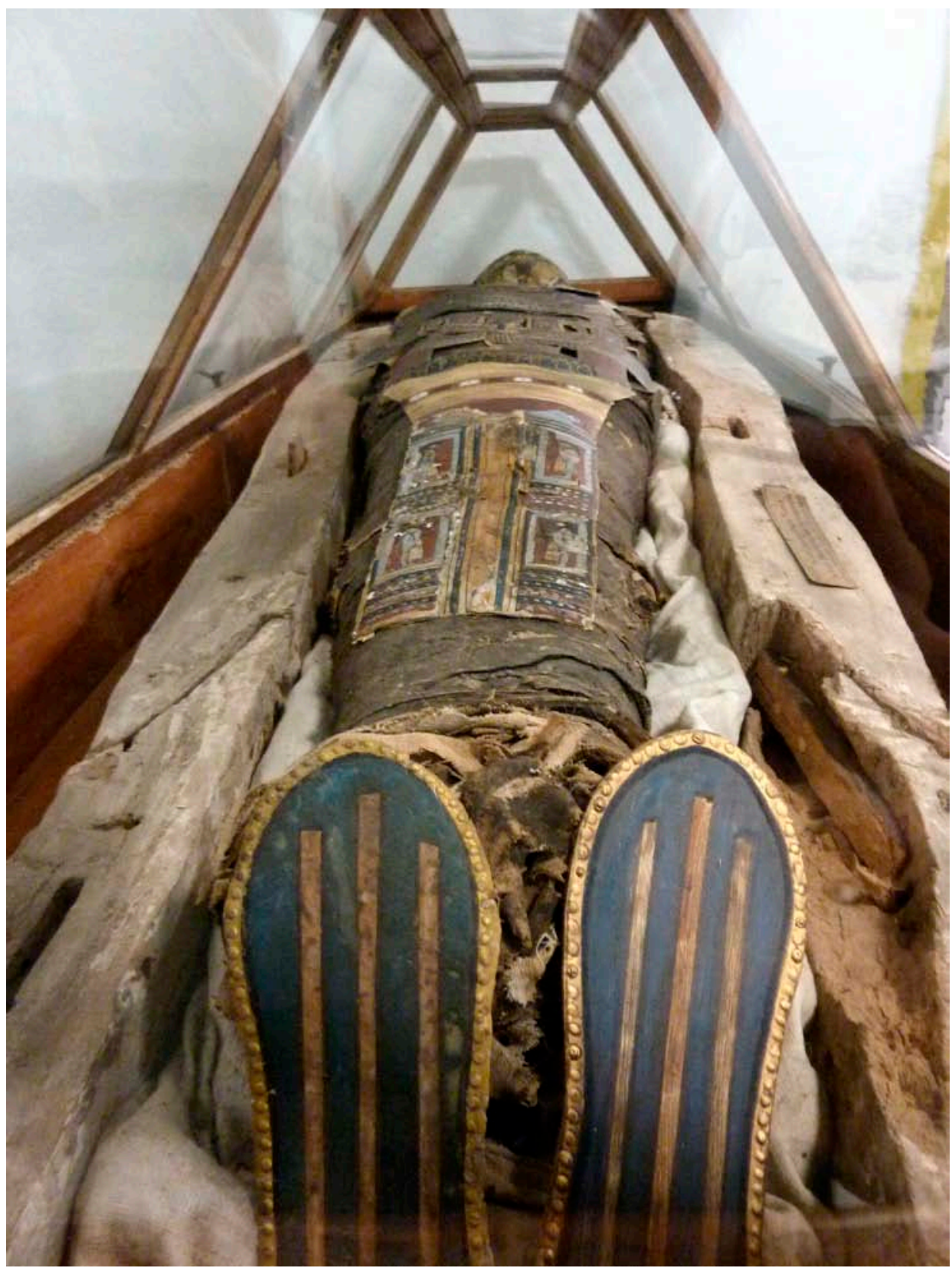

Abb. 4: Ägyptische Mumie des Academischen Museums. Heute Institut für Historische Anthropologie und Humanökologie der Universität Göttingen. Foto: D. Collet 
gebnissen. Niemand konnte ermitteln, woher die Mumie überhaupt stammte, so dass sie ihre Ergebnisse nicht mit bekannten Fakten in Beziehung setzten konnten. Zudem besaßen sie keinerlei Vergleichsmaterial. Bei der einzigen anderen „Mumie“ der Sammlung handelte es sich nur um ein europäisches Schaustellerstück - den weitgehend unverwesten Leichnam eines gewissen Conrad Schachtrupp aus Herzberg, der von einem Totengräber als Nebenerwerb in Wirtshäusern der Göttinger Umgebung vorgezeigt worden war. ${ }^{38}$ Auch als die Göttinger Professoren den Dänischen König später um ein weiteres Exemplar einer echten Mumie baten, war das Ziel nicht, an Vergleichsmaterial zu gelangen. Vielmehr war das erste Geschenk nunmehr so stark beschädigt, dass die Professoren es nicht länger unter dem Namen des königlichen Patrons ausstellen wollten. ${ }^{39}$

Diese Praxis des gutgläubigen Kopierens vermeintlicher britischer Vorbilder blieb nicht ohne Kritik. Mit deutlich größerer Skepsis begegneten ihnen Zeitgenossen, welche angelsächsische Sammlungen mit eigenen Augen gesehen hatten. So verfasste Georg Christoph Lichtenberg, der 1770 und 1775 nach England gereist war, kurz nach der Eröffnung des Göttinger Museums eine bitterböse Satire auf die britischen Museen. In seinem Text verzeichnete er Objekte eines Londoner Sammlers, der unschwer als der in Göttingen verehrte Sir Hans Sloane zu identifizieren war. Laut Lichtenberg enthielt diese Sammlung unter anderem eine

Bettstelle in Form eines Sarges [...] für Methodisten und Betschwestern, eine Suite von Kleidungsstücken für ein Kind mit zwei Köpfen und einen doppelte[n] Kinderlöffel für Zwillinge. ${ }^{40}$

Wie Lichtenberg befürchtete, führte die gewissenhafte Emulation des object turns der Royal Society durch seine Göttinger Kollegen zu vergleichbar ernüchternden Resultaten. Das Akademische Museum spiegelte keineswegs allein die Interessen der Gelehrten wider. Als öffentliche Sammlung reflektierte es ebenso sehr den Geschmack seiner vielen Besucher und Gönner sowie die Schwerpunkte des professionellen Kuriositätenhandels. Auch die gefeierte und bis heute berühmte Sammlung von Objekten der Reisen James Cooks enthielt nicht die von den Professoren ausdrücklich erbetenen Naturobjekte. Ihre Zusammensetzung illustrierte aber auch nicht bloß die repräsentativen Interessen ihres nominellen Spenders, des britischen Königs Georg III. In den

38 Gudrun Schwibbe, Wahrgenommen. Die sinnliche Erfahrung der Stadt, Münster 2002, S. 184. In einer weiteren Parallele zur Londoner Sammlung wurden auch in der Göttinger Sammlung Versuche mit vermeintlich giftigen, exotischen Objekten unternommen, die aber ebenso fruchtlos blieben. Frank William Peter Dougherty, The correspondence of Johann Friedrich Blumenbach, 4 Bde, Göttingen 2006-2012, hier Bd. 3, S. 176ff.

39 Eck, Mylius 1785 (wie Anm. 30), S. 43.

40 Wolfgang Promies (Hg.), Georg Christoph Lichtenberg. Schriften und Briefe, Bd. 3, München 1972, S. 451-457. 
ausgewählten exotischen Ethnographica kamen vielmehr die Vorlieben ganz anderer Akteure zum Ausdruck: die des mit dem Kauf beauftragten Kuriositätenhändlers George Humphreys, die der Virtuosi, für die Humphrey sonst tätig war, und die der Seeleute, welche die Objekte vor Ort gesammelt hatten (Abb. 5). ${ }^{41}$ In einigen Fällen dürften es auch die Gesammelten selbst gewesen sein, die die Zusammensetzung der Sammlung beeinflussten. So finden sich in der Göttinger Sammlung mehrere Schädel von neuseeländischen Maori. Diese weitgereisten Objekte könnte man leicht als eindrückliche Materialisierungen der Personalunion und der Netzwerke des britischen Empires interpretieren - zumal einer als Geschenk der Duchess of Northumberland, Charlotte Percy, und zwei andere über einen deutschen Handwerker am Hof in Windsor, Heinrich Ludwig Goertz, nach Göttingen gelangten. Tatsächlich wurden diese tätowierten, visuell beeindruckenden Schädel aber von den Maori in

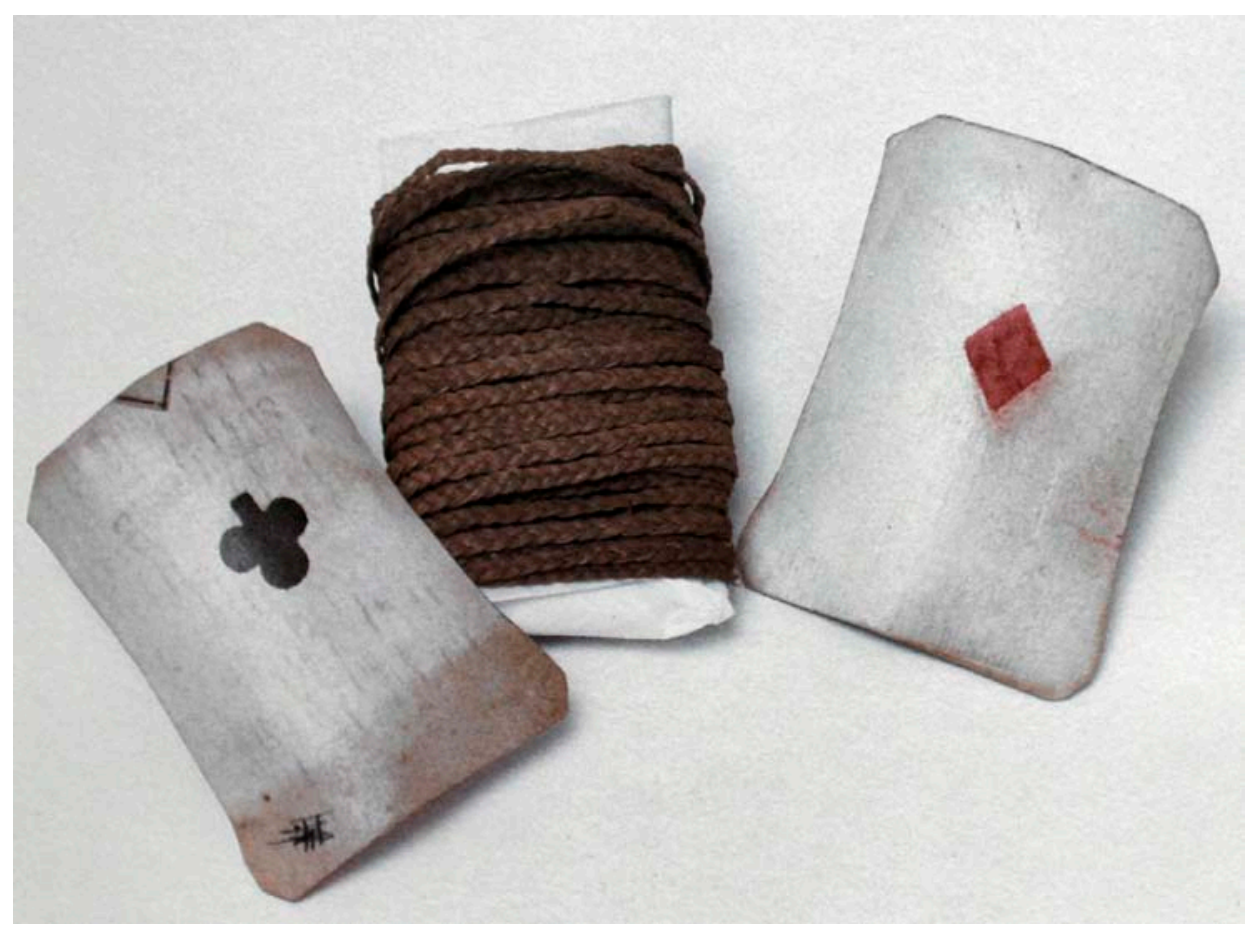

Abb. 5: Geflochtene Schnüre aus Tahiti. Kokosnussfasern auf Spielkarten der Seeleute der James Cook Expedition. Academisches Museum, heute Institut für Ethnologie, Universität Göttingen.

41 Humphreys selektive Vorlieben sind in seinem handschriftlichen Katalog der Sammlung dokumentiert, der heute im Institut für Völkerkunde in Göttingen verwahrt wird. Vgl. auch Thomas Nutz, Varietäten des Menschengeschlechts, Die Wissenschaften vom Menschen in der Zeit der Aufklärung, Köln u. a. 2009, S. $277-281$. 
großen Stückzahlen speziell für den europäischen Markt hergestellt. Sie nutzten das faszinierte Staunen der Europäer, um durch Handel an die begehrten Schusswaffen zu gelangen. In einigen Fällen dienten als Grundlage dieser vermeintlichen Exotika sogar die Schädel getöteter Europäer, die dazu extra eingefärbt und nachträglich tätowiert wurden. ${ }^{42}$ Ethnographische Forschungen waren an solchen, speziell für den europäischen Massengeschmack hergestellten Objekten, kaum durchführbar.

Stattdessen setzte sich auch in Göttingen zunächst wieder eine textbasierte Wissenspraxis durch, welche die Objekte nicht als Quellen sondern als Illustrationen verstand. Dies zeigt sich selbst dort, wo sich tatsächlich intensive, direkte Kontakte im Rahmen der Personalunion nachweisen lassen - etwa im Austausch zwischen dem Präsidenten der Royal Society, Joseph Banks, und dem Leiter des Göttinger Museums, Johann Friedrich Blumenbach. So brachte dieser Kontakt zwar eine große Anzahl von Objekten - vor allem menschliche Schädel - nach Göttingen, die Banks über sein weitgespanntes koloniales Netzwerk akquirieren konnte. Blumenbachs wirkmächtige Theorie über die Entstehung menschlicher Rassen war aber lange zuvor entstanden. ${ }^{43}$ Die Objekte dienten ihm nur mehr zur Illustration seiner zuvor gewonnenen Erkenntnisse, die er anstatt durch Autopsie durch die Lektüre von Reiseberichten gemacht hatte. Auch als Blumenbach 1781 daran ging seine einflussreiche Naturgeschichte zu illustrieren, ignorierte der Direktor des Museums seine eigene Sammlung und wandte sich stattdessen renommierten Büchern zu. Die Instruktionen an seinen Kupferstecher, Daniel Chodowiecki, lauteten daher:

1te Menschen-varietaet. eine Morgenländische Scene edelste Bildung! schlanker
Wuchs [....] Das ganze so viel [wie] möglich feinste Wollust athmend [...] Cos-
tüme etwa aus Niebuhrs Reisen [...]. 2te Menschen-Varietaet Chinesen [...] in
der Ferne ibre bizarren Lusthäusgen [...] wenns angeht auch etwa einige die Thee
trinken wobey etwan DuHaldes dexcription de la Chine nachzusehn. 4te Men-
schen-varietaet Brasilianer [...] wie der Virginier von Wencesl. Hollart (der auch
in Lavaters Physiognomik gut nachgestochen ist) [...] in der Ferne eine Hütte
[...]. Zur Vergleichung Cook's voyage round the world. (Abb. 6-8) ${ }^{44}$

Blumenbach hätte in der Göttinger Sammlung auf eine beachtliche Anzahl von Originalen aus den beschriebenen Regionen zurückgreifen können - Objekte, die oft

42 Gundolf Krüger, Mummified heads (mokomokai / upoko tui) from New Zealand in the Ethnographic Collection of Goettingen University, in: Dominik Collet / Marian Füssel / Roy McLeod (Hg.), The University of Things. History, practice, epistemology, Yearbook for European culture of science 9, (in Vorbereitung).

43 Nutz, 2009 (wie Anm. 41), S. 260f. und Céline Trautmann, Die Werkstatt Johann Friedrich Blumenbachs (1752-1840), in: Hans Erich Bödecker u.a. (Hg.), Die Wissenschaft vom Menschen in Göttingen um 1800. Wissenschaftliche Praktiken, institutionelle Geographie, europäische Netzwerke, Göttingen 2008, S. 213-254, hier S. $237 f$.

44 Blumenbach betonte, dass die Vignetten nicht etwa bloßzur Zierde des Buchs, sondern wesentlich nothwendig 
dem mit der Personalunion entstandenen Netzwerk entstammten. Aufgrund ihrer schlechten Dokumentation verließ er sich aber lieber auf die Autorität etablierter Bücher.

Es lassen sich also erstaunliche Parallelen zwischen den beiden Fallbeispielen beobachten: Die nahezu wortgleiche Propaganda für Sammlungen und Objektwissen als gelehrtes Reformprojekt, der jeweils vergebliche Versuch neue, dezidiert akademische Sammlungsformen zu etablieren, der Rückgriff auf die Technik des Fragebogens und des eigenen Experiments und schließlich die pragmatische Rückkehr zu traditionellen papiergebundenen Praktiken.

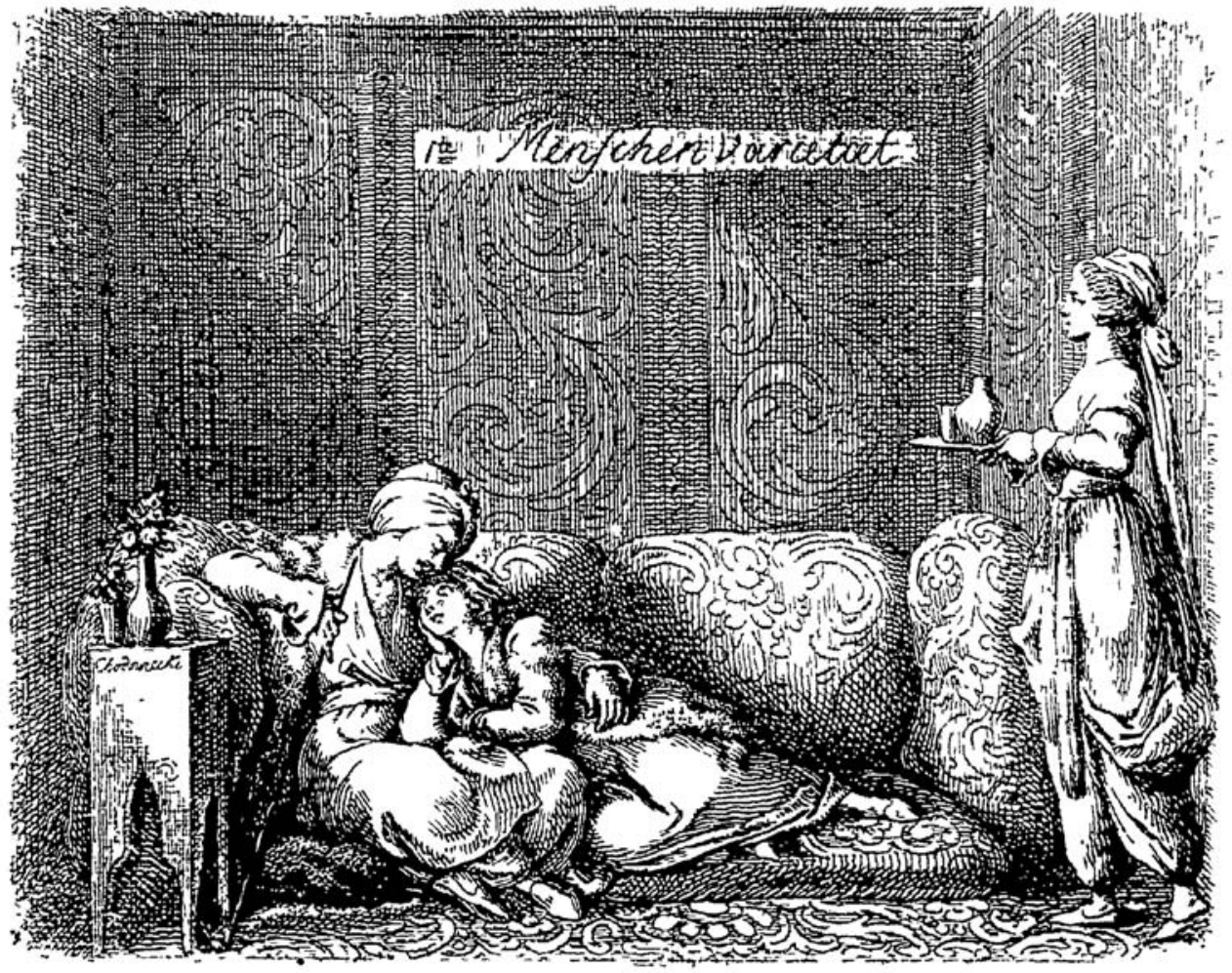

Abb. 6-8: Daniel Chodowiecki, Menschen-Varietäten, aus: Johann Friedrich Blumenbach, Beyträge zur Naturgeschichte. Göttingen 1790, SUB Göttingen

seien. Dougherty, 2006-2012 (wie Anm. 38), Bd. 1, S. 289-293. Die Illustrationen erschienen nach mehreren Korrekturen erstmals in: Johann Friedrich Blumenbach, Beyträge zur Naturgeschichte, Göttingen 1790. 

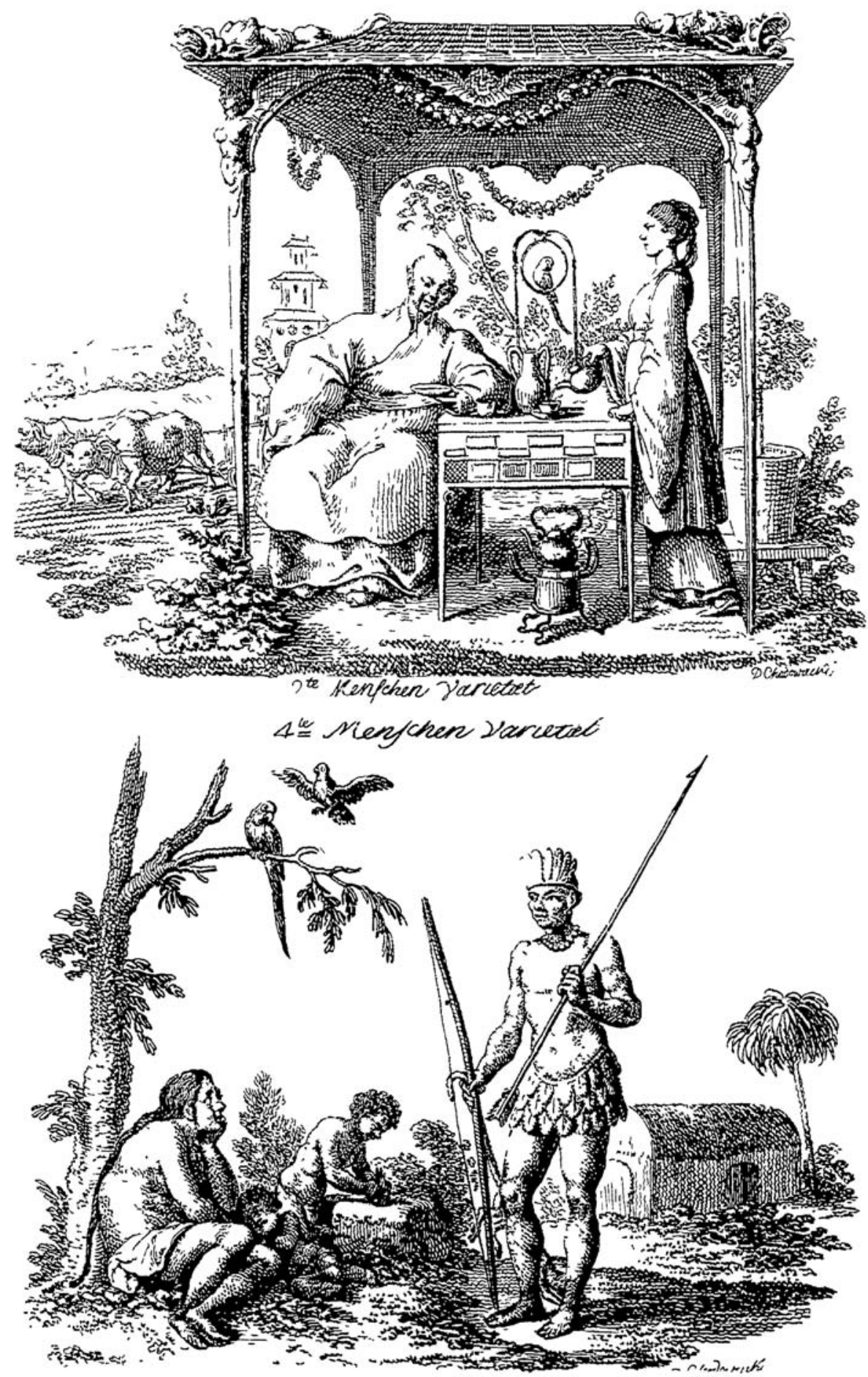


\section{Kreative Missverständnisse}

Es läge daher nah, das self-fashioning der Göttinger nach britischen Vorbildern als einen Fehlschlag oder zumindest als bedauernswertes Missverständnis zu deuten eine Interpretation, die sich nahtlos in das dominante Verständnis anglo-hannoveranischer Kontakte als historisch unbedeutend einfügen ließe und die Kurhannover als weitgehend passiven Empfänger britischer Ideen beschreibt. Man übersähe allerdings einen ganz zentralen Punkt: Die Göttinger Objekte begannen bald ein wirkmächtiges Eigenleben zu führen. Zwar hatten sie ihre ursprüngliche Aufgabe nicht erfüllt - ein Fehlschlag, der weniger in der räumlichen Distanz zwischen Hannover, Großbritannien und den Kolonien begründet lag, als in der sozialen Distanz zwischen Professoren und Sammlern. Sie übernahmen aber rasch ganz andere, gewichtige Aufgaben. Das Göttinger Academische Museum materialisierte die internationalen Netzwerke der jungen Universität - beispielsweise in der (wissenschaftlich zunächst enttäuschenden) James-Cook-Sammlung. ${ }^{45}$ Sie fungierte auch als Schauraum und als Erinnerungsort der Professoren und ihrer Erfolge. Ihr Direktor, Johann Friedrich Blumenbach, begriff aber, dass die Sammlung noch weiteres Potential hatte. Die Sammlung diente ihm dazu, die Institution Universität für zahlreiche neue Gruppen von Experten, Förderern und Naturliebhabern zu öffnen - eine Öffnung, die vielleicht ebenso viel zu Göttingens neuartigem Fokus in der Naturkunde beigetragen hat, wie die eigentlichen Forschungen ihrer Professoren. ${ }^{46}$

Anders als die Fellows der Royal Society vermochte es Blumenbach, die visuelle Evidenz der Exponate über seine Autorität als Professor mit akademischen Textpraktiken zu kombinieren. Während anderswo heftige Debatten darüber geführt wurden, ob Objekten oder Theorien im wissenschaftlichen Prozess der Vorrang gebühre - etwa zwischen dem Weltreisenden Georg Forster und dem Professor Immanuel Kant wusste Blumenbach beide Wissenskulturen geschmeidig miteinander zu verknüpfen. Seine geschickte Nutzung neuer materieller Ressourcen erlaubte es ihm eine Haltung einzunehmen, die man als „lächelndes Akzeptieren der Tradition“ bezeichnet hat. Im wissenschaftlichen Disput - etwa zur Natur der Fossilien oder zur Möglichkeit biologischer „Evolution“ - vermochte er dank seiner Objekte auch unorthodoxe Ergebnisse zu vertreten, ohne Widerspruch zu provozieren. ${ }^{47}$ Auf diese Weise erschloss Blu-

45 Das ethnographische Material der Cook-Forster-Sammlung war natürlich nur in den Augen der Zeitgenossen zunächst enttäuschend. Vgl. Dougherty, 2006-2012 (wie Anm. 38), Bd. 1, S. 327. Heute bildet ihre einzigartige materielle Überlieferung des Pazifiks und Nordamerikas eine unschätzbare Ressource ethnographischer Forschungen - ein Hinweis auf die häufigen Umdeutungen akademischer cinderella collections. Vgl. Krüger (wie Anm. 42) sowie Brigitta Hauser-Schäublin / Gundolf Krüger (Hg.), James Cook. Gaben und Schätze aus der Südsee. Die Göttinger Sammlung Cook / Forster, München / New York 1998.

46 Collet, 2012 (wie Anm. 24) und Trautmann, 2008 (wie Anm. 43).

47 Dougherty, 2006-2012 (wie Anm. 38), Bd. 3, S. 446 sowie Trautmann, 2008 (wie Anm. 43), S. 246. 
menbach der Universität neue, ehemals curieuse Wissensfelder. Exotika und Schädel, die sich in frühen Sammlungen großer Beliebtheit erfreuten, nutzte Blumenbach nun um die Völkerkunde und die physische Anthropologie als eigenständige universitäre Disziplinen zu fördern. ${ }^{48}$

Diese neue Verflechtung von Forschen und Zeigen positionierte die universitären Sammlungen bald im Zentrum des Prozesses akademischer Ausdifferenzierung. Sie half entstehende akademische Felder zu etablieren und naturalisierte zugleich deren Existenz. Viele akademische Fächer entstanden in Göttingen in enger Verbindung mit oder aufgrund der universitären Sammlungen: Neben der Völkerkunde und der Anthropologie zählten dazu auch die eng mit der Skulpturensammlung verbundene Kunstgeschichte, die in Göttingen ihren ersten deutschen Lehrstuhl bekam. Ganz ähnlich stützte der von Joseph Banks mit Pflanzen unterstützte „öconomische Garten“ später die Gründung der Forstwissenschaft, die wiederum die Loslösung der Biologie von der medizinischen Fakultät beförderte. ${ }^{49}$ Was als Fehlinterpretation früherer englischer Erfolge begonnen hatte, entwickelte sich rasch zu einem Katalysator neuer wissenschaftlicher Disziplinen und Praktiken, die schon bald weit über Göttingen hinaus kopiert wurden, bezeichnenderweise auch in Großbritannien. Als man in England zu Beginn des 19. Jahrhunderts daran ging die mittlerweile ins British Museum verlegte Sammlung der Royal Society neu zu ordnen, bediente man sich mit Carl (Charles) König eines Blumenbachschülers, der das Museum nach dem in der Göttinger Sammlung erprobten System neu strukturierte. Zwei weitere Blumenbachschüler, George Bellas Greenough und Carl Ludwig Giesecke, trugen später dazu bei, die objektgestützten Natur- und Geowissenschaften auch als Einzeldisziplinen in der britischen Wissenslandschaft zu etablieren. ${ }^{50}$

Diese Ergebnisse fügen sich in die Forschungen zu interkulturellen Kontakten in den letzten Jahren ein: Sie zeigen, dass Transferprozesse gerade nicht durch starre, vollständige Übernahmen gelingen, sondern dass der Austausch gerade da besonders rege ist, wo Ambivalenzen Transfers besonders attraktiv machen. ${ }^{51}$ Objekte sind aus dieser

48 Nutz, 2009 (wie Anm. 41), S. 275f.

49 Vgl. Collet, 2012 (wie Anm. 24), S. 48; sowie Schwibbe, 2002 (wie Anm. 38), S. 182. Zur engen Verbindung der Sammlungen mit der Genese der Fächer in Göttingen vgl.: Georg-August-Universität Göttingen (Hg.), Dinge des Wissens. Die Sammlungen, Museen und Gärten der Universität Göttingen, Göttingen 2012.

50 Thomas Biskup, The university of Göttingen and the Personal Union, 1737-1837, in: Simms und Riotte (Hg.), 2007 (wie Anm. 3) S. 150; Neil Chambers, Joseph Banks and the British Museum. The World of Collecting 1770-1830, London 2007, S. 34-43, 61-73 sowie Dougherty, 2006-2012 (wie Anm. 38), Bd. 3, S. 329. Vor der Neuordnung der Naturgeschichte im British Museum durch König beschwerten sich Besucher vom Kontinent: nothing is in order, every thing is out of its place; and this assemblage is rather an immense magazin [...] than a scientific collection. Hugh S. Torrens, Natural History in eighteenth-Century Museums in Britain, in: R.G.W. Anderson u.a. (Hg.), Englightening the British. Knowledge, discovery and the museum in the eighteenth century, London 2004, S. 81-91, hier S. 84.

51 Johannes Paulmann, Internationaler Vergleich und interkultureller Transfer. Zwei Forschungsansätze zur 
Perspektive deshalb außergewöhnlich effizient, weil sie besonders offen sind - wie sich etwa an der Göttinger Cook-Sammlung beobachten lässt, deren Objekte von den Zeitgenossen zugleich als Symbole für das Britische Empire, als Forschungsobjekte und als unterhaltende Kuriositäten verstanden wurden.

\section{Im Netz der Dinge}

Zusammenfassend lässt sich feststellen: Die Wissenspraxis des Sammelns konstituiert ein Netz der Dinge, ein Handlungsgeflecht, das eine sehr heterogene Gruppe von Akteuren miteinander in Beziehung bringt. Für viele Gelehrte des 17. und 18. Jahrhunderts lag die Herausforderung dieser Form des Kontakts nicht in der Überwindung der geographischen Distanz zwischen Hannover, England und der kolonialen Welt, sondern in der sozialen Distanz zwischen den Beteiligten - dies galt insbesondere in der fragilen, ganz besonders auf Stand und Stellung bedachten Welt der Gelehrten. Als Göttinger Professoren nach Reformmodellen Ausschau hielten, blickten sie zunächst auf den neuen Kommunikations- und Wissensraum, der mit der Personalunion entstanden war - Haller, Michaelis und Blumenbach reisten selbst nach London. Für Blumenbach war sein Englandbesuch im Jahr 1793 die einzige Auslandsreise überhaupt. Ihre gutwillige Übernahme der vermeintlichen Erfolge der Royal Society lässt sich als Missverständnis interpretieren, das auch auf den Wunsch zurückging, innerhalb der neuen Allianz von Hannover und Großbritannien tätig zu werden. Allerdings initiierte dieses Missverständnis unerwartete Innovationen. Die Übersetzung eines britischen Sammlungsmodells in den Rahmen einer deutschen Universität kreierte eine Wissenskultur, die neue Disziplinen, neue Expertengruppen und neue Formen von Soziabilität, Evidenz und Beweis etablierte - Bereiche, die in der Wissenschaftsgeschichte mittlerweile als zentrale Felder des wissenschaftlichen Prozesses verstanden werden..$^{52}$ Als Kontaktraum für gelehrte Geselligkeit trugen die Sammlungen wesentlich dazu bei, neuen Erkenntnissen zum Durchbruch zu verhelfen, wissenschaftliche Codes zu verbreiten und entstehende Wissensfelder institutionell zu konsolidieren. Über die Visualisierung von Evidenz am materiellen Zeugen konnte akademisches Wissen leichter zwischen Lehrern und Schülern, Sammlern und Besuchern, Kuratoren und Amateuren zirkulieren.

europäischen Geschichte des 18. bis 20 Jahrhunderts, in: Historische Zeitschrift 267 (1998), S. 649-685; Michael Werner / Bénédicte Zimmermann, Vergleich, Transfer, Verflechtung. Der Ansatz der Histoire croisée und die Herausforderung des Transnationalen, in: Geschichte und Gesellschaft 28 (2002), S. 607-636.

52 Vgl. etwa: Steven Shapin, Never Pure. Historical Studies of Science as if It Was Produced by People with Bodies, Situated in Time, Space, Culture and Society, and Struggling for Credibility and Authority, Baltimore 2010. 
Es ist daher keineswegs zufällig, dass viele dieser unintendierten Neuschöpfungen im Laufe des 19. Jahrhunderts mit Blumenbachs Schülern wieder zurück auf die britischen Inseln gelangten und sich die Göttinger Taxonomien und Wissensfelder im British Museum, den britischen wissenschaftlichen Gesellschaften und dem modernen Universitätscurriculum in beiden Territorien etablierten. Die Dynamik solcher kreativen Missverständnisse ist im Feld der Wissenschaft besonders sichtbar und anhand von Objekten auch materiell direkt nachvollziehbar. Sie markiert aber eine Form des unintendierten Austauschs, die auch in anderen Bereichen häufig und wirkmächtig gewesen sein könnte - Bereiche, in denen Kulturtransfers bisher allzu rasch ausgeschlossen worden sind. ${ }^{33}$

53 Zur möglichen Neubewertung von Austauschprozessen in den Feldern der Kunst, der Wissenschaft, der Diplomatie, des Militärs sowie der Ökonomie vgl. Hölscher/Schlitte 2014; Horst Carl / Uwe Ziegler (Hg.), ,In unserer Liebe nicht glücklich'. Kultureller Austausch zwischen Großbritannien und Deutschland 1770-1840, Göttingen 2014; Katja Lembke (Hg.), Hannovers Herrscher auf Englands Thron 1714-1837, Dresden 2014. 